\title{
FINITE GROUPS WHICH ARE PRODUCTS OF PAIRWISE TOTALLY PERMUTABLE SUBGROUPS
}

\author{
by A. BALLESTER-BOLINCHES, M. C. PEDRAZA-AGUILERA \\ and M. D. PÉREZ-RAMOS
}

(Received 18th November 1996)

\begin{abstract}
Finite groups which are products of pairwise totally permutable subgroups are studied in this paper. The $\mathcal{F}$-residual, $\mathcal{F}$-projectors and $\mathcal{F}$-normalizers in such groups are obtained from the corresponding subgroups of the factor subgroups under suitable hypotheses.
\end{abstract}

1991 Mathematics subject classification: 20F17, $20 \mathrm{D} 40$.

Throughout the paper we consider only finite groups. The well-known fact that the product of two normal supersoluble subgroups is not in general supersoluble makes interesting the study of factorized groups whose subgroup factors are connected by certain permutability properties. In this context, Asaad and Shaalan [1] proved the following result:

Let $G=G_{1} G_{2}$ be a group which is the product of two supersoluble subgroups $G_{1}$ and $G_{2}$. If every subgroup of $G_{1}$ is permutable with every subgroup of $G_{2}$ (we say then that $G_{1}$ and $G_{2}$ are totally permutable subgroups of $G$ ), then $G$ is supersoluble.

Later, Maier [8] proves that this property is not only true for the class of all supersoluble groups but also for all saturated formations $\mathcal{F}$ containing $\mathcal{U}$, the class of supersoluble groups. Two different extensions to this result are made in [3] and [5]. On the one hand, the first and third authors show that the above result extends to nonsaturated formations containing $\mathcal{U}$.

Theorem A [3]. Let $\mathcal{F}$ be a formation containing $\mathcal{U}$. Suppose that the group $G=G_{1} G_{2}$ is the product of the totally permutable subgroups $G_{1}$ and $G_{2}$. If $G_{1}, G_{2} \in \mathcal{F}$. then $G \in \mathcal{F}$.

On the other hand, a generalization of Maier's result to an arbitrary finite number of factors is given in [5] by Carocca in the following way. 
Theorem B [5]. Let $G=G_{1} G_{2} \ldots G_{r}$ be a group such that $G_{1}, G_{2}, \ldots, G_{r}$ are pairwise totally permutable subgroups of $G$. Let $\mathcal{F}$ be a saturated formation which contains $\mathcal{U}$. If for all $i \in\{1,2, \ldots, r\}$ the subgroups $G_{i}$ are in $\mathcal{F}$, then $G \in \mathcal{F}$.

Also in this context, the following result is proved in [2]:

Theorem $\mathbf{C}$ [2]. Let $\mathcal{F}$ be a formation of soluble groups such that $\mathcal{U} \subseteq \mathcal{F}$. If $G=H K$ is the product of the totally permutable subgroups $H$ and $K$, then $G^{\mathcal{F}}=H^{\mathcal{F}} K^{\mathcal{F}}$. Here $G^{\mathcal{F}}$ denotes the $\mathcal{F}$-residual of $G$, that is, the smallest normal subgroup of $G$ with quotient in $\mathcal{F}$.

Our main goal in this paper is to take these studies further. In fact we prove that Carocca's result can be extended to non-saturated formations containing $\mathcal{U}$. We also study the behaviour of $\mathcal{F}$-residuals, $\mathcal{F}$-projectors and $\mathcal{F}$-normalizers, where $\mathcal{F}$ is a saturated formation, in such factorized groups.

Lemma 1. Let $\mathcal{F}$ denote a formation containing $\mathcal{U}$. Consider a group $G=G_{1} G_{2} \ldots G_{r}$ such that $G_{1}, G_{2}, \ldots, G_{r}$ are pairwise totally permutable subgroups of $G$. Then $G_{i}^{\mathcal{F}}$ is a normal subgroup of $G$ for all $i \in\{1,2, \ldots, r\}$.

Proof. It is a direct consequence of Lemma 3 of [2].

Theorem 1. Let $G=G_{1} G_{2} \ldots G_{r}$ be a group such that $G_{1}, G_{2}, \ldots, G_{r}$ are pairwise totally permutable subgroups of $G$. Let $\mathcal{F}$ be a formation such that $\mathcal{U} \subseteq \mathcal{F}$. If $G_{i} \in \mathcal{F}$ for all $i \in\{1,2, \ldots, r\}$, then $G$ belongs to $\mathcal{F}$.

Proof. Assume that the result is false and let $G$ be a counterexample with $|G|+\left|G_{1}\right|+\ldots+\left|G_{r}\right|$ minimal. Then $r>2$ and by Theorem B, there exists $i \in\{1,2, \ldots, r\}$ such that $G_{i}$ does not belong to $\mathcal{U}$. Without loss of generality, we suppose $i=1$. Then $G_{1}^{U} \neq 1$. By [2, Corollary], the subgroup $K=G_{2} \ldots G_{r}$ centralizes $G_{1}^{u}$. Hence $G_{1}^{u}$ is a normal subgroup of $G$. Moreover $G_{1}^{u}$ centralizes $\left\langle K^{G}\right\rangle$, the normal closure of $K$ in $G$. On the other hand, $G_{1}=G_{1}^{U} A$, for an $\mathcal{U}$-projector $A$ of $G_{1}$. Consider now $Z=\left[\left\langle K^{G}\right\rangle\right] G_{1}$, the semidirect product of $\left\langle K^{G}\right\rangle$ and $G_{1}$ with respect to the action by conjugation. By [3, Lemma 1], $G$ is isomorphic to a quotient of $Z$. We prove that $Z$ is an $\mathcal{F}$-group. It is clear that $G_{1}^{u}$ is a normal subgroup of $Z$ because it is centralized by $\left\langle K^{G}\right\rangle$. Moreover $Z /\left(G_{1}^{U}\right)$ is isomorphic to $\left[\left\langle K^{G}\right\rangle\right]\left(A /\left(A \cap G_{1}^{u}\right)\right)$, which is a quotient of $\left[\left\langle K^{G}\right\rangle\right] A$. Next we see that $\left[\left\langle K^{G}\right\rangle\right] A \in \mathcal{F}$. From the fact that $G_{1}^{u}$ centralizes $K$, one can easily deduce $\left\langle K^{G}\right\rangle=\left\langle K^{A}\right\rangle$. A new application of $[3$, Lemma 1] yields the existence of an epimorphism $\Psi$ from $\left[\left\langle K^{A}\right\rangle\right] A$ onto $K A$. So $\left[\left\langle K^{A}\right\rangle\right] A / \operatorname{Ker} \Psi$ is isomorphic to $K A$, which is a product of pairwise totally permutable $\mathcal{F}$-subgroups. Notice that $|K A|+|A|+\left|G_{2}\right|+\ldots+\left|G_{r}\right|<|G|+\left|G_{1}\right|+\ldots+\left|G_{r}\right|$. So $K A \in \mathcal{F}$ by the minimal choice of $\left(G, G_{1}, \ldots, G_{r}\right)$. Now, since $\left[\left\langle K^{A}\right\rangle\right] A /\left\langle K^{A}\right\rangle$ is isomorphic to $A \in \mathcal{U} \subseteq \mathcal{F}$, we have that $\left[\left\langle K^{A}\right\rangle\right] A /(\operatorname{Ker} \Psi \cap A) \in \mathcal{R}_{0} \mathcal{F}=\mathcal{F}$. But $(\operatorname{Ker} \Psi) \cap\left\langle K^{A}\right\rangle=1$ by [3, Lemma 1]. Consequently, $\left[\left\langle K^{A}\right\rangle\right] A \in \mathcal{F}$ and then $Z / G_{1}^{U} \in \mathcal{F}$. Moreover, $Z /\left\langle K^{G}\right\rangle$ is isomorphic to 


\section{FINITE GROUPS OF PAIRWISE TOTALLY PERMUTABLE SUBGROUPS 569}

$G_{1} \in \mathcal{F}$. Therefore $Z \simeq Z /\left(\left(K^{G}\right\rangle \cap G_{1}^{u}\right) \in \mathcal{R}_{0} \mathcal{F}=\mathcal{F}$ and $G \in \mathcal{F}$, a contradiction.

Our next theorem shows that the converse of Maier's result remains true in the case of a product of pairwise totally permutable subgroups and formations containing $\mathcal{U}$ which are either saturated or soluble. We need first a preliminary lemma.

Lemma 2. Let $\mathcal{F}$ be a formation such that $\mathcal{U} \subseteq \mathcal{F}$ and let $G=G_{1} G_{2} \ldots G_{r}$ be a product of pairwise totally permutable subgroups $G_{1}, \ldots, G_{r}$. If $G_{2}, G_{3}, \ldots, G_{r}, G$ are $\mathcal{F}$-groups, then $G_{1} \in \mathcal{F}$.

Proof. It is clear that we can assume $G_{1}^{U} \neq 1$ and $G_{1}=G_{1}^{U} U$, for an $\mathcal{U}$-projector $U$ of $G_{1}$. By [3, Lemma 1$], G_{1}$ is an epimorphic image of $C=\left[G_{1}^{u}\right] U$, the semidirect product of $G_{1}^{u}$ and $U$ with respect to the conjugation action. So it is enough to prove that $C \in \mathcal{F}$. Notice that from Lemma $1, G_{1}^{U}$ is a normal subgroup of $G$. Moreover $G=G_{1}^{u}(U K)$, where $K=G_{2} G_{3} \ldots G_{r}$. Also by Lemma 1 of [3] there exists an epimorphism $\alpha: X \rightarrow G$ with $(\operatorname{Ker} \alpha) \cap G_{1}^{u}=1$, where $X=\left[G_{1}^{u}\right](U K)$. In particular, $X /(\operatorname{Ker} \alpha) \simeq G \in \mathcal{F}$. On the other hand, $X / G_{1}^{u} \simeq U K$. Notice now that $U K$ is a product of pairwise totally permutable $\mathcal{F}$-subgroups. Consequently, by Theorem $1, U K \in \mathcal{F}$. Now $X / G_{1}^{u} \in \mathcal{F}$ and from here $X /\left(G_{1}^{U} \cap \operatorname{Ker} \alpha\right) \simeq X \in \mathcal{F}$. By [2, Corollary], we know that $\left\langle K^{X}\right\rangle=\left\langle K^{U}\right\rangle$, the normal closure of $K$ in $K U$. In particular, $\left\langle K^{X}\right\rangle$ is contained in $K U$. This implies that $\left\langle K^{X}\right\rangle \cap G_{1}^{u}=1$. Moreover $X /\left(K^{X}\right\rangle \simeq\left[G_{1}^{u}\right]\left(U /\left(\left\langle K^{X}\right\rangle \cap U\right)\right)$ and so $\left[G_{1}^{u}\right] U /\left(\left\langle K^{X}\right\rangle \cap U\right)$ belongs to $\mathcal{F}$. This means that $\left[G_{1}^{u}\right] U /\left(\left\langle K^{X}\right) \cap G_{1}^{u} \cap U\right) \simeq\left[G_{1}^{u}\right] U \in$ $\mathcal{R}_{0} \mathcal{F}=\mathcal{F}$. Consequently, $C=\left[G_{1}^{u}\right] U \in \mathcal{F}$ and the lemma is proved.

Theorem 2. Let $\mathcal{F}$ be a saturated formation containing the class $\mathcal{U}$ and let $G=G_{1} G_{2} \ldots G_{r}$ be the product of the pairwise totally permutable subgroups $G_{1}, G_{2}, \ldots, G_{r}$. If $G \in \mathcal{F}$, then $G_{i} \in \mathcal{F}$ for all $i \in\{1,2, \ldots, r\}$.

Proof. Arguing by induction on the order of $G$, we can assume $G$ has a unique minimal normal subgroup $N$ and $G_{i} N / N \in \mathcal{F}$ for all $i \in\{1,2, \ldots, r\}$. By Lemma 1(a) of [5], there exists $i \in\{1,2, \ldots, r\}$ such that $N \leq G_{i}$. We can assume without loss of generality that $N \leq G_{1}$. Take now $j \in\{1,2, \ldots, r\}$ with $j \neq 1$. Using the fact that $G_{j} N / N \in \mathcal{F}$ we have $G_{j}=T_{j}\left(G_{j} \cap N\right)$, where $T_{j}$ is an $\mathcal{F}$-projector of $G_{j}$. Notice now that $T_{j} \leq G_{j}$ and $G_{j} \cap N \leq G_{1}$. Moreover by [5, Lemma $\left.1(\mathrm{~b})\right], G_{j} \cap G_{1} \leq F\left(G_{j} G_{1}\right) \in \mathcal{U}$. Hence $G_{j}$ is the product of two totally permutable $\mathcal{F}$-subgroups. By Theorem $1, G_{j} \in \mathcal{F}$. Consequently $G_{j} \in \mathcal{F}$ for all $j \in\{2, \ldots, r\}$. Applying Lemma 2, we conclude that $G_{1} \in \mathcal{F}$.

Theorem 3. Let $\mathcal{F}$ be a formation such that $\mathcal{U} \subseteq \mathcal{F} \subseteq \mathcal{S}$ (here $\mathcal{S}$ is the class of soluble groups). Let $G=G_{1} G_{2} \ldots G_{r}$ be the product of the pairwise totally permutable subgroups $G_{1}, G_{2}, \ldots, G_{r}$. If $G \in \mathcal{F}$, then $G_{i} \in \mathcal{F}$ for every $i \in\{1,2, \ldots, r\}$.

Proof. We argue by induction on $|G|+\left|G_{1}\right|+\left|G_{2}\right|+\ldots+\left|G_{r}\right|$. We can assume that there exists $i \in\{1,2, \ldots, r\}$ such that $G_{i}$ does not belong to $\mathcal{U}$. Suppose without loss of 
generality that $i=r$. Since $\mathcal{U}$ is a saturated formation, we have that $G_{r}^{u}$ is not contained in $\Phi\left(G_{r}\right)$, the Frattini subgroup of $G_{r}$. Moreover, since $G \in \mathcal{F}$, we have that $G$ is soluble. In particular, $G_{r}$ is soluble and then the Fitting subgroup of $G_{r}^{u} /\left(G_{r}^{u} \cap \Phi\left(G_{r}\right)\right)$ denoted $F /\left(G_{r}^{u} \cap \Phi\left(G_{r}\right)\right)$, is different from 1. Since $F /\left(G_{r}^{u} \cap \Phi\left(G_{r}\right)\right)$ is nilpotent, we have that $F$ is nilpotent by [4, Theorem 3.7]. Notice that $F \unlhd G_{r}^{u} \unlhd G$. Hence $F$ is subnormal in $G$. Then $F$ is contained in $F(G)$. On the other hand $F$ is a normal subgroup of $G_{r}$ which is not contained in $\Phi\left(G_{r}\right)$. So there exists a maximal subgroup $M$ of $G_{r}$ such that $F$ is not contained in $M$. This implies $G_{r}=F M$ and $G=F\left(M G_{1} G_{2} \ldots G_{r-1}\right)=$ $F(G)\left(M G_{1} G_{2} \ldots G_{r-1}\right)$. Hence $M G_{1} G_{2} \ldots G_{r-1}$ is a subgroup of $G$ supplementing a nilpotent normal subgroup of $G$. By $[6, I V, 1.14]$ we have that $M G_{1} G_{2} \ldots G_{r-1} \in \mathcal{F}$. Moreover $J=M G_{1} G_{2} \ldots G_{r-1}$ is a product of pairwise totally permutable subgroups such that $|J|+|M|+\left|G_{1}\right|+\ldots+\left|G_{r-1}\right|<|G|+\left|G_{1}\right|+\ldots+\left|G_{r}\right|$. This implies by induction that $G_{1}, G_{2}, \ldots, G_{r-1}$ are $\mathcal{F}$-groups. Applying now Lemma 2 we conclude that $G_{r} \in \mathcal{F}$.

Remark 1. Theorems 2 and 3 are not true without assuming that either $\mathcal{F}$ is a saturated formation of $\mathcal{F}$ is contained in $\mathcal{S}$. The group $H=S L(2,5)$ has a normal subgroup of order 2, say $Z(H)=F(H)=\phi(H)$ with $H / Z(H) \simeq A_{5}$. Take $G=X \times Y$ with $X \simeq H \simeq Y$ and denote $D=\langle x y\rangle$ where $x$ and $y$ are the central involutions of $X$ and $Y$ respectively. Consider the group $G / D$, that is, the central product of $X$ and $Y$ (see [6, A, 19] for further details). Let $\mathcal{F}=\mathcal{Q R}_{0}(G / D)$ be the formation generated by the group $G / D$ and $\mathcal{F} \circ \mathcal{U}$ the formation product of $\mathcal{F}$ and $\mathcal{U}$ (recall that $\mathcal{U} \subseteq \mathcal{F} \circ \mathcal{U}$ ). Notice that $G / D$ is a perfect group. Hence $(G / D)^{\mathcal{F} O \mathcal{U}}=(G / D)^{\mathcal{F}}=1$ and $G / D \in \mathcal{F} \circ \mathcal{U}$. However $H$ does not belong to $\mathcal{F} \circ \mathcal{U}$ (see [7,2.3]). So $G / D$ is an $\mathcal{F} \circ \mathcal{U}$-group which is a totally permutable product with factors $X$ and $Y$ not in $\mathcal{F} \circ \mathcal{U}$.

Theorem 4. Let $\mathcal{F}$ be a formation containing $\mathcal{U}$ such that $\mathcal{F}$ is either saturated or $\mathcal{F} \subseteq \mathcal{S}$. If $G=G_{1} G_{2} \ldots G_{r}$ is the product of the pairwise totally permutable subgroups $G_{i}$ for $i \in\{1,2, \ldots r\}$, then $G^{\mathcal{F}}=G_{1}^{\mathcal{F}} G_{2}^{\mathcal{F}} \ldots G_{r}^{\mathcal{F}}$.

Proof. We use induction on the order of $G$. We can assume $G^{\mathcal{F}} \neq 1$, otherwise the result follows from Theorems 2 and 3 . Notice that the hypotheses in the theorem are inherited by the quotient group $G / G^{\mathcal{F}}$. So we have by induction that $G_{i} G^{\mathcal{F}} / G^{\mathcal{F}}$ is an $\mathcal{F}$ group for every $i \in\{1,2, \ldots, r\}$. In particular, $G_{i}^{\mathcal{F}} \leq G^{\mathcal{F}}$ for each $i \in\{1,2, \ldots, r\}$. On the other hand we know that $G_{i}^{\mathcal{F}}$ is a normal subgroup of $G$ for $i \in\{1,2, \ldots, r\}$ by Lemma 1. Therefore $G_{1}^{\mathcal{F}} G_{2}^{\mathcal{F}} \ldots G_{r}^{\mathcal{F}}$ is a normal subgroup of $G$ contained in $G^{\mathcal{F}}$. Denote $K=G_{1}^{\mathcal{F}} G_{2}^{\mathcal{F}} \ldots G_{r}^{\mathcal{F}}$. If $K=1$, we have $G_{i} \in \mathcal{F}$ for each $i \in\{1,2, \ldots, r\}$ and the result follows from Theorem 1. So we can assume $K$ is non-trivial. Consider now $N$ a minimal normal subgroup of $G$ contained in $K$. Now the result is true for the quotient group $G / N$ and we have $G^{\mathcal{F}}=K N=K$.

Remark 2. Conditions on $\mathcal{F}$ in Theorem 4 are also necessary. Consider the group $G=X \times Y$ and the formation $\mathcal{F} \circ \mathcal{U}$ as in Remark 1 . As $H$ does not belong to $\mathcal{F} \circ \mathcal{U}$ and $H \in \mathcal{Q}(G)$, we have that $G$ is not an $(\mathcal{F} \circ \mathcal{U})$-group. On the other hand, 


\section{FINITE GROUPS OF PAIRWISE TOTALLY PERMUTABLE SUBGROUPS 571}

$G / D \in \mathcal{F} \circ \mathcal{U}$. Moreover $D$ is a minimal normal subgroup of $G$. Then $G^{\mathcal{F} d}=D$. Consider $H / Z(H) \simeq A_{5} \in \mathcal{Q}(G / D) \subseteq \mathcal{F} \circ \mathcal{U}$. Also $Z(H)$ is a minimal normal subgroup of $H$. Thus $H^{\mathcal{F} \alpha}=Z(H)$. Hence $X^{\mathcal{F} \alpha U} \times Y^{\mathcal{F} \alpha}=\langle x\rangle \times\langle y\rangle \neq\langle x y\rangle=D=G^{\mathcal{F} \alpha U}$.

Finally we prove that both $\mathcal{F}$-projectors and $\mathcal{F}$-normalizers have a good behaviour in groups factorized as the product of pairwise totally permutable subgroups.

Theorem 5. Let $\mathcal{F}$ be a saturated formation containing $\mathcal{U}$ and let $G=G_{1} G_{2} \ldots G_{r}$ be the product of the pairwise totally permutable subgroups $G_{i}$ for $i \in\{1,2, \ldots, r\}$. If $A_{1}, A_{2}, \ldots, A_{r}$ are $\mathcal{F}$-projectors of $G_{1}, G_{2}, \ldots, G_{r}$ respectively, then the product $A_{1} A_{2} \ldots A_{r}$ is an $\mathcal{F}$-projector of $G$.

Proof. We prove the result by induction on the number of factors. If $r=2$, it follows from [2, Theorem B]. Assume it is true for a number of factors $t<r$. Suppose the theorem is false when $t=r$ and choose $G$ of minimal order satisfying this condition. We can then assume $G_{i} \neq 1$ for each $i \in\{1,2, \ldots, r\}$. By [5, Lemma 1(a)], there exists $i \in\{1,2, \ldots, r\}$ such that $G_{i}$ contains a minimal normal subgroup of $G, N$ say. Assume without loss of generality that $i=1$. Now the minimality of $G$ yields that $\left(A_{1} N / N\right)\left(A_{2} N / N\right) \ldots\left(A_{r} N / N\right)=\left(A_{1} A_{2} \ldots A_{r}\right) N / N$ is an $\mathcal{F}$-projector of $G / N$. Denote $C=\left(A_{1} A_{2} \ldots A_{r}\right) N$ and assume $C$ is a proper subgroup of $G$. We have then $C$ as the product of the pairwise totally permutable subgroups $A_{1} N, A_{2}, \ldots, A_{r}$. Moreover applying [6, III, 3.14 and 3.18] we have that $A_{1}$ is an $\mathcal{F}$-projector of $A_{1} N$. Therefore, by the minimality of $G, A_{1} A_{2} \ldots A_{r}$ is an $\mathcal{F}$-projector of $C$. Applying now [6, III, 3.7], we have that $A_{1} A_{2} \ldots A_{r}$ is an $\mathcal{F}$-projector of $G$, a contradiction. Consequently $G=\left(A_{1} A_{2} \ldots A_{r}\right) N$. By Theorem $1, A_{1} A_{2} \ldots A_{r} \in \mathcal{F}$. Hence $G^{\mathcal{F}} \leq N$ and $G^{\mathcal{F}}=1$ or $G^{\mathcal{F}}=N$. If $G^{\mathcal{F}}=1$ the result follows from Theorem 2. So we may assume $G^{\mathcal{F}}=N$. Consider now an $\mathcal{F}$-maximal subgroup $U$ of $G$ containing $A_{1} A_{2} \ldots A_{r}$. By [6, III, 3.14 and 3.18], $U$ is an $\mathcal{F}$-projector of $G$. Moreover $U$ is the product of the pairwise totally permutable subgroups $A_{1}(U \cap N), A_{2} \ldots, A_{r}$. By Lemma 2, we have that $A_{1}(U \cap N)$ belongs to $\mathcal{F}$. But $A_{1}$ is an $\mathcal{F}$-maximal subgroup of $G_{1}$. This implies that $U \cap N \leq A_{1}$ and $U=A_{1} A_{2} \ldots A_{r}$ is an $\mathcal{F}$-projector of $G$.

Theorem 6. Let $G=G_{1} G_{2} \ldots G_{r}$ be a soluble group which is the product of the pairwise totally permutable subgroups $G_{1}, G_{2}, \ldots G_{r}$. Let $\mathcal{F}$ be a saturated formation containing $\mathcal{U}$. If $A_{1}, A_{2}, \ldots, A_{r}$ are $\mathcal{F}$-normalizers of $G_{1}, G_{2}, \ldots G_{r}$ respectively, then $A_{1} A_{2} \ldots A_{\text {r }}$ is an $\mathcal{F}$-normalizer of $G$.

Proof. We argue by induction on $|G|+\left|G_{1}\right|+\ldots+\left|G_{r}\right|$. By Theorem 1, we can assume that there exists $i \in\{1,2, \ldots, r\}$ such that $G_{i}$ does not belong to $\mathcal{F}$. Suppose $i=1$. Since $\mathcal{F}$ is a saturated formation, $G_{1}^{\mathcal{F}}$ is not contained in $\Phi\left(G_{1}\right)$, the Frattini subgroup of $G_{1}$. Hence $T /\left(G_{1}^{\mathcal{F}} \cap \Phi\left(G_{1}\right)\right)$, the Fitting subgroup of $G_{1}^{\mathcal{F}} /\left(G_{1}^{\mathcal{F}} \cap \Phi\left(G_{1}\right)\right)$ is non-trivial (notice that $G$ is a soluble group). Now, by [4, Theorem 3.7], $T$ is nilpotent. Consequently $T$ is a nilpotent normal subgroup of $G_{1}$ not contained in $\Phi\left(G_{1}\right)$. This implies the existence of a maximal subgroup $M$ of $G_{1}$ such that $T$ is not contained in 
$M$. Therefore $G_{1}=T M=G_{1}^{\mathcal{F}} M=F\left(G_{1}\right) M$ and $M$ is $\mathcal{F}$-critical in $G_{1}$. Now by [6, V, 3.2 and 3.7], every $\mathcal{F}$-normalizer of $M$ is an $\mathcal{F}$-normalizer of $G$ and they are conjugated subgroups in $G$. We can then assume $A_{1} \leq M$. Then $G=T\left(M G_{2} G_{3} \ldots G_{r}\right)=$ $F(G)\left(M G_{2} G_{3} \ldots G_{r}\right)=G^{\mathcal{F}}\left(M G_{2} G_{3} \ldots G_{r}\right)$ (notice that, by Theorem 2, $G_{1}^{\mathcal{F}} \leq G^{\mathcal{F}}$ ). If we assume $G=M G_{2} \ldots G_{r}$, then $M, G_{2}, \ldots, G_{r}$ are pairwise totally permutable subgroups of $G$ and $|G|+|M|+\left|G_{2}\right|+\ldots+\left|G_{r}\right|<|G|+\left|G_{1}\right|+\left|G_{2}\right|+\ldots\left|G_{r}\right|$. By induction we have $A_{1} A_{2} \ldots A_{r}$ is an $\mathcal{F}$-normalizer of $G$, and the theorem is true. So we can assume $M G_{2} G_{3} \ldots G_{r}$ is a proper subgroup of $G$. Then $M G_{2} G_{3} \ldots G_{r}$ is an $\mathcal{F}$-critical maximal subgroup of $G$ and each $\mathcal{F}$-normalizer of $M$ is an $\mathcal{F}$-normalizer of $G$. By induction, $A_{1} A_{2} \ldots A_{r}$ is an $\mathcal{F}$-normalizer of $M G_{2} G_{3} \ldots G_{r}$. Hence, $A_{1} A_{2} \ldots A_{r}$ is an $\mathcal{F}$-normalizer of $G$.

Acknowledgements. The research of the first and third authors is supported by Proyecto PB 94-0965 of DGICYT, MEC, Spain. Part of the research of the second author has been done in the Johannes Gutenberg-Universität Mainz under the support of Beca Bancaixa-Europa III ciclo (Doctorado). Also the work of this author is supported by Beca Predoctoral de formación del Personal Investigador de la Consellería de Educación y Ciencia of Valencia (Spain).

\section{REFERENCES}

1. M. ASAAD and A. ShaAlan, On the supersolvability of finite groups, Arch. Math. 53 (1989), 318-326.

2. A. Ballester-Bolinches, M. C. Pedraza-Aguilera and M. D. Pérez-Ramos, On finite products of totally permutable groups, Bull. Austral. Math. Soc. 53 (1996), 441-445

3. A. Ballester-Bolinches and M. D. Pérez-Ramos, A Question of R. Maier concerning formations, J. Algebra 182 (1996), 738-747.

4. A. Ballester-Bolinches and M. D. Pérez-Ramos, On $\mathcal{F}$-subnormal subgroups and Frattini-like subgroups of a finite group, Glasgow Math. J. 36 (1994), 241-247.

5. A. Carocca, A note on the product of $\mathcal{F}$-subgroups in a finite group, Proc. Edinburgh Math. Soc. 39 (1996), 37-42.

6. K. DOERK and T. HAWKeS, Finite soluble groups (Walter De Gruyter, Berlin-New York, 1992).

7. K. Doerk and T. Hawkes, On the residual of a direct product, Arch. Math. (Basel) 30 (1978), 458-468.

8. R. MAIER, A completeness property of certain formations, Bull. London Math. Soc. 24 (1992), 540-544.

\section{Departament D'Algebra UNIVERSITAT DE VALÈNCIA \\ C/ DOCTOR MOLINER 50 46100 BURJASSOT (VALĖNCIA) SPAIN}

